\title{
Handover checklist: testing a standardization process in an Italian hospital
}

This article was published in the following Dove Press journal:

Risk Management and Healthcare Policy

22 May 2017

Number of times this article has been viewed

\author{
Davide Ferorelli' \\ Teresa Giandola ${ }^{2}$ \\ Mariangela Laterza ${ }^{2}$ \\ Biagio Solarino ${ }^{2}$ \\ Angela Pezzolla ${ }^{3}$ \\ Fiorenza Zotti ${ }^{2}$ \\ Alessandro Dell'Erba' \\ 'Interdisciplinary Department of \\ Medicine, ${ }^{2}$ Section of Legal Medicine, \\ ${ }^{3}$ Department of Emergency and Organ \\ Transplantation, University of Bari, \\ Bari, Italy
}

Objectives: This study aimed to standardize and rationalize the handover, a critical and essential moment in common health care practices, through the realization of an efficient and standardized checklist, which could be used daily to ensure complete, thorough and effective handover. The principal purpose of the implementation of the handover is to reduce errors due to superficial and insufficient communication.

Methods: The "operative group" defined the phases to the realization of the delineated aims: at first, the direct observation and the consequent realization of a handover checklist model and then, the experimental phases (trials). The handover checklist model was used for a month and it was daily and duly completed by the doctors who took part in the trial. To prove the success of the study, three questionnaires were distributed on different occasions.

Results: Analyzing the answers to the questionnaires, the importance of the handover has come to light and that for the most part, the doctors consider it an essential and irreplaceable moment in daily health care work. Moreover, it became obvious that the use of the handover checklist guaranteed a considerable improvement in the traditional handover in terms of security, completeness, care continuity and clarity. The handover checklist was completely appreciated by the majority of the participant doctors who agree with the definitive introduction of it in their unit. Conclusions: Our study indicated the consistency of the handover checklist as an instrument to implement the handover and, indirectly, to improve the quality of the care.

Keywords: clinical risk management, handover checklist, health care workers training

\section{Introduction}

In health care, the handover is referred to as the practice of exchanging a patient's clinical information with other health care professionals during shift rotation or patient transfer. The handover represents a high-risk event in which errors commonly occur which can harm patients. ${ }^{1}$ In addition to the exchange of clinical information, the handover implies a transfer of responsibility for the patient's well-being between health care workers. Therefore, the handover is a critical and essential moment in common health care practices.

Despite the importance of this every day process, a lack of communication prevails and has become common across the wards; this has a negative impact on patient safety and management. ${ }^{2}$ Consequently, it is necessary to pay serious attention to this process and to the implications of health care worker handover.

\section{High 5s project}

With the aim of preventing errors due to inaccurate handover, we have taken part in the "High 5s Project". This is a global patient safety initiative to facilitate the development,
Correspondence: Davide Ferorelli Istituto di Medicina Legale, Policlinico Bari, Italy

Tel +39805478290

Email davideferorelli@gmail.com
Risk Management and Healthcare Policy 2017:10 87-93 
implementation and evaluation of standard operating protocols in order to achieve measurable, significant and sustainable reductions in challenging patient safety problems. ${ }^{3}$

The handover is essential because it provides accurate information about the patient and allows the shift workers to provide responsible health care for the patient. This is done in accordance with the current core competence in nursing and medical education which includes quality improvement, patient-centered care, teamwork and collaboration, using evidence-based practices, safety and informatics. ${ }^{4}$

Furthermore, the handover guarantees health care continuity, cooperative learning, group collaboration improvements, respect, confidence, continuous development and sharing. Handover tools are increasingly used today to provide an effective and standardized platform for information exchange. ${ }^{5}$

At times, health care workers do not pay the correct amount of attention to the handover process, and this may lead to consequences for the patient. In such cases, there is a lack of communication and comprehension among health care professionals. This can lead to an increased amount of adverse events and embarrassing situations among health care professionals because the clinicians involved in the handover do not actively listen and instead end up "doing" everything at the same time. ${ }^{6}$ As is evident in the literature, a lack of communication leads to consequences with regard to decision-making and impairs the health care system; this may result in repetition of examinations which have already been performed.

The standardization of the handover is an extremely crucial aspect in the health care system because it is a highrisk point in clinical care, which, if mismanaged, can lead to adverse events or near misses. ${ }^{7}$ Despite the effects of an insufficient handover, there are not any scientific publications which highlight factors which should be part of this system, such as health care worker performance, procedures and efficacy of structured interventions.

A number of documents and tools finalized to make the handover more effective have been elaborated worldwide, but yet, to this day, a univocal model does not exist and for this reason, the choice of an appropriate and every day working situation-suitable tool is indispensable.

One of the tools which risk managers use to implement and standardize the handover is the checklist, which has a standard structure derived from existing practices. ${ }^{8}$ It guarantees procedure safety and avoids potential mistakes which may cause serious complications, and can reduce the mortality rate.
This is a pilot study about the implementation of an efficient tool for an effective handover which could be used daily in health care settings to ensure complete and effective communication between the incoming and the outgoing health care workers; the used tool is standardized and rationalized. ${ }^{9}$

The instrument is a standardized checklist which increases the quantity and the quality of transmitted medical information. ${ }^{10}$ The perception of clinicians on applicability of these standardized tools has been also investigated to evaluate the feasibility of the standard use of the tool.

Nowadays, the checklist is recognized as an important health support tool, and it is useful in ensuring that important and essential information is not omitted during the handover. Consequently, we can avoid the loss of information during shift change. ${ }^{11}$ The principal purpose of the implementation of the handover is to reduce errors due to superficial and insufficient communication, so the patient's complications, morbidity and mortality rate decrease. ${ }^{12}$

\section{Methods}

The study refers to a handover checklist which is useful for the doctors' handover. This checklist has been created and updated by the risk management staff after a literature review and in this article, we present our experience about the validation of the checklist.

An "operative group" coordinated by the Risk Management Coordinator and the Coordinators of the Videolaparoscopic Surgery Unit and the Orthopedic and Traumatology Unit of the General Hospital of the Public Health Corporation of Bari ( 1500 beds), was set up; this group carried out the first phase observation and planning. The team members are physician specialists in Phorensic Medicine, Surgery, and Orthopedic and Traumatology. We chose the Videolaparoscopic Surgery Unit and the Orthopedic and Traumatology Unit because in the past other risk management activities were carried out in this setting.

The group defined four necessary phases to the realization of the delineated aims: the first phase for direct observation and planning (February 2016), the second phase for staff education (March 2016) and to understand the purpose, impact and experience of the handover from the staff perspective. ${ }^{13}$ The third (April 2016) and fourth (May and June 2016) were the experimental phases (trials).

The first phase of the pilot project included the direct observation of the Videolaparoscopic Surgery Unit (14 beds, $\sim 1000$ surgical procedures for each year). The execution of the morning handover at 8:30 and the afternoon one at 14:00 were observed. 
During this phase, it was immediately noted that the information was usually transcribed in a disorganized and nonfunctional way, giving a disorderly, dispersive and unmethodical overall view.

On the basis of the data collected during the first phase of direct observation, it was possible to create an initial model of the handover checklist (Table S1). This model presents a well-organized and well-structured configuration. Each item in the list corresponds to the information which has to be communicated.

The new form gives a more immediate and clearer vision of the patient care priorities, reducing the duration of the handover and as is well known with the use of a written checklist, increasing significantly the overall number of items handed over. ${ }^{14}$

This model of handover checklist represents an individual tool which has to be filled in for each patient and it has to be inserted into the medical record after patient discharge. The items were assembled in five macro-categories: vital signs, diet, examinations, patient condition and therapy/treatment.

There are boxes, near the list of items, which have to be ticked each time the information is properly communicated during the handover. There is also a special area, near the boxes, where any important alert can be written, such as an alternated vital sign as well as the schedule of a particular examination.

The first trial attempt in the operative unit of the Videolaparoscopic Surgery failed. Thanks to the suggestions and criticisms received it has been possible to modify the initial checklist model and to create a better one, which is more appropriate for the necessities of the unit. For this reason, in the new checklist model, some of the items have been replaced by others ( Table S2).

To avoid further failure, a multiple-choice questionnaire was distributed in the Orthopedic and Traumatology Unit at the beginning of the new trial phase. The questionnaire's aim was to assess general knowledge about the handover and its effectiveness in the Orthopedic and Traumatology Unit (30 beds, 4000 surgical procedures each year). The questionnaire was filled in by 12 people, ten of whom ( $83 \%$ ) were trainee doctors.

To prove the success of the study, another multiple-choice questionnaire was distributed on two different occasions: before the induction of the new handover checklist model and after its adoption into the trial phase. The questionnaire was filled in by $83 \%$ of the doctors and examined aspects of handover security, completeness, continuity and clarity (Table S3). At the end of the trial phase, a new questionnaire was distributed to the trainee doctors, who mostly did the everyday handover, working in the Orthopedic and Traumatology Unit. It was composed of four open questions (Table S4). The questionnaire was only filled in by $25 \%$ of the doctors.

The first attempt at the use of the handover checklist application was made in the Videolaparoscopic Surgery Unit in April 2016. In this period, the handover checklist was not used at all, leading to the failure of the initial trial phase. The medical professionals of this unit were skeptical about the use of the new tool. The justifications for the group's insufficient compliance were exaggerated workload because the checklist is individual and it has to be filled in for every single patient, difficulty finding it in the medical record (already full of documents), lack of specific information, difficult approach, difficult to understand, and lack of instruction document.

This failure was assessed and an attempt was made to learn something useful from an analysis of the mandatory data. ${ }^{15}$ Some changes were suggested through team consultation. This was appropriate because a systematic approach to interventions through facilitating opportunities for communication openness, cooperation and exchange of ideas between health care workers is needed to improve the level of patient safety culture. ${ }^{16}$

The second attempt to use the new handover checklist was made at the Orthopedic and Traumatology Unit (20 beds). The Orthopedic and Traumatology Unit involved the unit director, 12 trainee doctors and ten doctors. In this unit, the doctors used to do the handover twice a day, at 8:00 and at 20:00; the information used to be communicated verbally between a trainee doctor and a specialized doctor, and they used to write down the most important notes on a blank page.

During the second trial phase, the handover checklist was daily and duly completed by the doctors. The study was approved by the Head of the Risk Management Unit of the Bari Policlinico General Hospital and because personal data were not collected, informed consent was not requested or obtained.

\section{Results}

Analyzing the answers to the first questionnaire (Table 1), the importance of the handover has come to light and that for the most part, the doctors consider it an essential and irreplaceable moment in daily health care work. This is in agreement with the literature which considers the standardization of the handover process necessary to ensure continuity and safety of care. ${ }^{17}$

Sixty percent of the doctors who filled out the questionnaire consider themselves satisfied with the way they generally perform the handover in the unit where they work, 
Table I Answers to the first questionnaire

\begin{tabular}{|c|c|c|c|c|c|}
\hline & Totally agree \% & Agree, \% & Uncertain, \% & Slightly agree, \% & Disagree, \% \\
\hline $\begin{array}{l}\text { Do you think handover is an essential moment in health } \\
\text { care common practice? }\end{array}$ & 10 & 60 & 20 & 10 & - \\
\hline $\begin{array}{l}\text { Do you think the orthopedic unit gives the right } \\
\text { importance to the handover? }\end{array}$ & 10 & 60 & 20 & 10 & - \\
\hline $\begin{array}{l}\text { Do you consider yourself satisfied with the way the } \\
\text { handover is performed in your unit? }\end{array}$ & - & 60 & 30 & 10 & - \\
\hline $\begin{array}{l}\text { Do you think some of the medical errors made in your } \\
\text { unit are due to a lack in the flow of information? }\end{array}$ & 20 & 40 & 30 & 10 & - \\
\hline Do you consider it useful to improve the handover process? & 30 & 30 & 30 & 10 & - \\
\hline $\begin{array}{l}\text { Do you think the checklist could be an adequate tool to } \\
\text { improve the handover process? }\end{array}$ & 30 & 30 & 30 & 10 & - \\
\hline
\end{tabular}

Table 2 Answers to the first questionnaire (pre-/post-checklist adoption)

\begin{tabular}{|c|c|c|c|c|c|}
\hline & Totally agree & Agree & Uncertain & Slightly agree & Disagree \\
\hline \multirow[t]{2}{*}{ Security } & Pre-adoption: $10 \%$ & Pre-adoption: $50 \%$ & Pre-adoption: $30 \%$ & Pre-adoption: $10 \%$ & Pre-adoption: $0 \%$ \\
\hline & Post-adoption: $30 \%$ & Post-adoption: $70 \%$ & Post-adoption: $0 \%$ & Post-adoption: 0\% & Post-adoption: $0 \%$ \\
\hline \multirow[t]{2}{*}{ Completeness } & Pre-adoption: $10 \%$ & Pre-adoption: 30\% & Pre-adoption: 50\% & Pre-adoption: $0 \%$ & Pre-adoption: $10 \%$ \\
\hline & Post-adoption: $20 \%$ & Post-adoption: $80 \%$ & Post-adoption: $0 \%$ & Post-adoption: $0 \%$ & Post-adoption: $0 \%$ \\
\hline \multirow[t]{2}{*}{ Continuity } & Pre-adoption: $0 \%$ & Pre-adoption: $20 \%$ & Pre-adoption: $50 \%$ & Pre-adoption: $10 \%$ & Pre-adoption: $10 \%$ \\
\hline & Post-adoption: $10 \%$ & Post-adoption: $90 \%$ & Post-adoption: 0\% & Post-adoption: $0 \%$ & Post-adoption: $0 \%$ \\
\hline \multirow[t]{2}{*}{ Clarity } & Pre-adoption: $0 \%$ & Pre-adoption: $10 \%$ & Pre-adoption: $10 \%$ & Pre-adoption: $60 \%$ & Pre-adoption: $10 \%$ \\
\hline & Post-adoption: $80 \%$ & Post-adoption: $20 \%$ & Post-adoption: $0 \%$ & Post-adoption: 0\% & Post-adoption: $0 \%$ \\
\hline
\end{tabular}

despite the fact that $60 \%$ of them think that some of the medical errors, made by the unit where they work, are probably due to a lack of flow of information. For this reason, most of the doctors state that it is useful to standardize the handover and $60 \%$ of them think the checklist is an excellent tool which could be used to reach this goal.

In analyzing the answers to the second questionnaire (Table S3), it became obvious that the use of the handover checklist guaranteed a considerable improvement in the traditional handover in terms of security, completeness, care continuity and clarity (Table 2).

After the trial phase, an open-question questionnaire (Table S4) was distributed to better identify the strengths and weaknesses of the handover checklist; the questionnaire also took into account the suggestions of the health care workers who took part in the study, in line with the new need for organizational transformation with a focus on staff experience and leadership development. ${ }^{18}$

All doctors who filled out the questionnaire state that the handover checklist allows them to obtain a snapshot of patient care priorities and to have a more complete picture of the patient's anamnesis. Importantly, this allows, especially in an emergency, for the health care worker to have comprehensive knowledge about the patient and what he/she needs. Thus, the doctor is facilitated in taking the best and safest decisions for the patient's care.
Some of the doctors also asserted that the use of the handover checklist leads to a more accurate and correct handover, reducing omission in information during coworkers' communication.

The doctors who completed the questionnaire agreed that the principal negative aspect to the handover checklist is that it takes a long time to fill in for any single patient. All things considered, $66 \%$ of the health care professionals who took part in the project agreed to adopting the handover checklist as a permanent and final tool to standardize the handover.

\section{Conclusion}

This study represents a first attempt to standardize the handover, an important and crucial moment in common health care practice. The main goal is the creation and validation of a useful tool which could guarantee an efficient handover and which could be used on a daily basis in any ward of the hospital. To achieve this aim, a checklist was used which led to a more complete, faster and more precise handover. The adoption of the handover checklist allowed, indirectly, for further aims to be achieved, such as increased safety for the patient, thanks to the improvement in coworkers' communication during the handover, and leads to the prevention of medical errors caused by an insufficient and inadequate handover. 
The presence of the handover checklist in the medical record of the patient represents an efficient proof of the correct health care practice the patient benefitted from during their stay in hospital. It, therefore, follows that health care professionals obtain greater protection in the face of potential legal action.

The results have been based on the answers to the questionnaires filled out by professionals of the Orthopedic and Traumatology Unit. From analysis of the results, it has been possible to conclude that the use of the handover checklist has led to a considerable improvement in the traditional handover in terms of security, completeness, continuity and clarity.

Moreover, the handover checklist was fully appreciated by the majority of the participant doctors who agreed with the definitive introduction of it in their unit. Despite these results, there was a poor response to the open-question questionnaire leading to a lack of statistically valid data. The fact that the same group of people fully completed the multiple-choice questionnaire, and not the open-question one, underlines again that the convenience and practicality of a list makes the checklist more efficient than an unstructured tool which has to be completed at the complete discretion of the doctor.

Despite the handover checklist obtaining a positive outcome, the participant doctors have doubts about the time that it takes to complete a checklist for any single patient. For this purpose, the adoption of a tool including information about all of the unit's patients on a single form, which has to be filled out daily, could be useful. In this way, the doctor would have a detailed and accurate picture of the patient and, at the same time, a global picture of all the unit's patients. This could lead to a considerable reduction in the handover duration, plus a reduction in the documents which have to be consulted out of necessity.

\section{Disclosure}

The authors report no conflicts of interest in this work.

\section{References}

1. Young JQ, van Dijk SM, O'Sullivan PS, Custers EJ, Irby DM, Ten Cate O. Influence of learner knowledge and case complexity on handover accuracy and cognitive load: results from a simulation study. Med Educ. 2016;50(9):969-978.
2. Saunsbury E, Howarth G. Improving communication between phlebotomists and doctors: a quality improvement project. BMJ Qual Improv Rep. 2016;5(1): u206305.w4089.

3. Leotsakos A, Zheng H, Croteau R, et al. Standardization in patient safety: the WHO High 5s project. Int J Qual Health Care. 2014;26(2): $109-116$.

4. Mako T, Svanäng P, Bjerså K. Patients' perceptions of the meaning of good care in surgical care: a grounded theory study. BMC Nurs. 2016;15:47.

5. Alhamid SM, Lee DX, Wong HM, et al. Implementing electronic handover: interventions to improve efficiency, safety and sustainability. Int J Qual Health Care. 2016;28(5):608-614.

6. Calleja P, Aitken L, Cooke M. Staff perceptions of best practice for information transfer about multitrauma patients on discharge from the emergency department: a focus group study. J Clin Nurs. 2016;25(19-20):2863-2873.

7. Brook J, Amaro Calcia M. Improving the quality of handover in a liaison psychiatry team. BMJ Qual Improv Rep. 2016;5(1): u206492.w3442.

8. Redley B, Bucknall TK, Evans S, Botti M. Inter-professional clinical handover in post-anaesthetic care units: tools to improve quality and safety. Int J Qual Health Care. 2016;28(5):573-579.

9. Foronda C, MacWilliams B, McArthur E. Interprofessional communication in healthcare: An integrative review. Nurse Educ Pract. 2016;19:36-40.

10. Salzwedel C, Mai V, Punke MA, Kluge S, Reuter DA. The effect of a checklist on the quality of patient handover from the operating room to the intensive care unit: a randomized controlled trial. J Crit Care. 2016;32:170-174.

11. LeBlanc J, Donnon T, Hutchison C, Duffy P. Development of an orthopedic surgery trauma patient handover checklist. Can J Surg. 2014;57(1):8-14.

12. Dojmi Di Delupis F, Pisanelli P, Di Luccio G, et al. Communication during handover in the pre-hospital/hospital interface in Italy: from evaluation to implementation of multidisciplinary training through high-fidelity simulation. Intern Emerg Med. 2014;9(5):575-582.

13. Bruton J, Norton C, Smyth N, Ward H, Day S. Nurse handover: patient and staff experiences. Br J Nurs. 2016;25(7):386-390, 392-393.

14. Salzwedel C, Bartz HJ, Kühnelt I, et al. The effect of a checklist on the quality of post-anaesthesia patient handover: a randomized controlled trial. Int J Qual Health Care. 2013;25(2):176-181.

15. Buja A, Saieva AM, Vinelli A, et al. Adverse event reporting and patient safety at a University Hospital: Mapping, correlating and associating events for a data-based patient risk management. Int J Risk Saf Med. 2016;28(3):163-170.

16. Wami SD, Demssie AF, Wassie MM, Ahmed AN. Patient safety culture and associated factors: A quantitative and qualitative study of healthcare workers' view in Jimma zone Hospitals, Southwest Ethiopia. BMC Health Serv Res. 2016;16:495.

17. Smeulers M, Vermeulen H. Best of both worlds: combining evidence with local context to develop a nursing shift handover blueprint. Int $J$ Qual Health Care. 201628(6):749-757.

18. Nkomazana O, Mash R, Phaladze N. Understanding the organisational culture of district health services: Mahalapye and Ngamiland health districts of Botswana. Afr J Prim Health Care Fam Med. 2015;7(1):907. 


\section{Supplementary materials}

Table SI Initial model of handover checklist

\begin{tabular}{|c|c|c|c|c|}
\hline & AGE: & & & \\
\hline \multicolumn{5}{|l|}{ F/M } \\
\hline \multirow{2}{*}{\multicolumn{5}{|c|}{$\begin{array}{l}\text { Diagnosis: } \\
\text { DATE Recovery: }\end{array}$}} \\
\hline & & & & \\
\hline \multicolumn{5}{|l|}{ DATE Surgery: } \\
\hline \multirow[t]{6}{*}{ VITAL SIGNS: } & & $\square$ & $\square$ & $\square$ \\
\hline & $\mathrm{CF}$ & 口 & 口 & $\square$ \\
\hline & RR & $\square$ & 口 & 口 \\
\hline & AP & $\square$ & $\square$ & $\square$ \\
\hline & $\mathrm{T}^{\circ} \mathrm{C}$ & - & 口 & 口 \\
\hline & $\mathrm{SO} 2$ & व & 口 & ㅁ \\
\hline DIET: & & $\square$ & $\square$ & $\square$ \\
\hline EXAMINATION: & & $\square$ & $\square$ & $\square$ \\
\hline Lab & & 口 & $\square$ & 口 \\
\hline Instrumental & & ㅁ & 口 & 口 \\
\hline \multirow[t]{4}{*}{ PATIENT CONDITION: } & & $\square$ व & $\square$ व & $\square$ व \\
\hline & Feces & 口 & 口 & 口 \\
\hline & Allergies & 口 & $\square$ & $\square$ \\
\hline & Diuresis & - & $\square$ & 口 \\
\hline \multicolumn{5}{|l|}{ Medication } \\
\hline & Drainage & - & $\square$ & $\square$ \\
\hline & Access & 口 & 口 & 口 \\
\hline \multirow[t]{4}{*}{ Therapy: } & & $\square$ & $\square$ & $\square$ \\
\hline & Dialysis & ㅁ & $\square$ & ㅁ \\
\hline & Consultation & ㅁ & $\square$ & 口 \\
\hline & Transfusion & ㅁ & 口 & ㅁ \\
\hline INCOMING: & & & & \\
\hline OUTGOING: & & & & \\
\hline
\end{tabular}

Abbreviations: $F$, female; $M$, male; $C F$, cardiac frequency; $R R$, respiratory rate; $A P$, arterial pressure; $T^{\circ} \mathrm{C}$, temperature; SO2, oxygen saturation.

Table S2 New model of handover checklist

\begin{tabular}{|c|c|c|c|c|}
\hline \multirow{3}{*}{$\begin{array}{l}\text { MR/MRS: } \\
\text { Diagnosis: } \\
\text { DATE recovery: }\end{array}$} & \multirow[t]{3}{*}{ AGE: F/M } & DAY & \multicolumn{2}{|c|}{ NIGHT } \\
\hline & & & & \\
\hline & & & & \\
\hline \multicolumn{5}{|l|}{ DATE surgery: } \\
\hline VITAL SIGNS: & & $\square$ & $\square$ & \\
\hline \multirow[t]{2}{*}{ EXAMINATION: } & Lab & $\square \quad \square$ & $\square$ & $\square$ \\
\hline & Instrumental & $\square$ & & $\square$ \\
\hline \multirow[t]{4}{*}{ PATIENT CONDITION: } & Allergies & $\square \quad \square$ & $\square$ & $\square$ \\
\hline & Hemotransfusion & $\square$ & & $\square$ \\
\hline & Diuresis & $\square$ & & $\square$ \\
\hline & Discharge & $\square$ & & $\square$ \\
\hline \multirow[t]{2}{*}{ Therapy: } & Medical & $\square \quad \square$ & $\square$ & $\square$ \\
\hline & Consultation & $\square$ & & $\square$ \\
\hline \multirow[t]{2}{*}{ OUTGOING: } & MD & & & \\
\hline & Trainee doctor & & & \\
\hline \multirow[t]{2}{*}{ INCOMING: } & MD & & & \\
\hline & Trainee doctor & & & \\
\hline
\end{tabular}

Abbreviations: F, female; M, male; MD, Medical Doctor. 
Table S3 Questionnaire distributed before and after the induction of the new handover checklist

I. The incoming doctor receives, through the handover, a complete picture of the patient which guarantees the safety of the patient himself.

Totally agree Agree Uncertain Slightly agree Disagree

$\square \quad \square \quad \square \quad \square \quad \square$

2. The incoming doctor completely understands, through the handover, what are the priorities which have to be considered.

Totally agree Agree Uncertain Slightly agree Disagree

3. The incoming doctor receives, through the handover, a complete picture of the actual patient condition.

Totally agree Agree Uncertain Slightly agree Disagree

$\square \quad \square \quad \square \quad \square \quad \square$

4. The information shared during the handover, guarantees the continuity of the patient's care

Totally agree Agree Uncertain Slightly agree Disagree

$\begin{array}{lllll}\square & \square & \square & \square & \square\end{array}$

5. The handover is performed in a clear and univocal way

Totally agree Agree Uncertain Slightly agree Disagree

$\begin{array}{llll}\square & \square & \square & \square\end{array}$

\section{Table S4 Open-question questionnaire}

I. Thinking about the handover checklist adopted in the trial phase: what are the positive aspects? Did the handover improve?

2. What are the negative aspects?

3. Do you have any ideas to improve the handover checklist?

4. Do you think that the handover checklist could be a permanent tool to perform the handover? If no, why?

Risk Management and Healthcare Policy

\section{Publish your work in this journal}

Risk Management and Healthcare Policy is an international, peer-reviewed, open access journal focusing on all aspects of public health, policy, and preventative measures to promote good health and improve morbidity and mortality in the population. The journal welcomes submitted papers covering original research, basic science, clinical and epidemiological

\section{Dovepress}

studies, reviews and evaluations, guidelines, expert opinion and commentary, case reports and extended reports. The manuscript management system is completely online and includes a very quick and fair peerreview system, which is all easy to use. Visit http://www.dovepress.com/ testimonials.php to read real quotes from published authors. 